\title{
BMJ Open Eversion technique versus conventional endarterectomy with patch angioplasty in carotid surgery: protocol for a systematic review with meta-analyses and trial sequential analysis of randomised clinical trials
}

\author{
Martijn S. Marsman (D) , Jorn Wetterslev (D) , ${ }^{2}$ Patrick W.H.E. Vriens, ${ }^{3}$ \\ Ronald L.A.W. Bleys, ${ }^{4}$ Abdelkarime Kh. Jahrome, ${ }^{5}$ Frans L. Moll, ${ }^{6}$ Frederik Keus, ${ }^{7}$ \\ Giel G. Koning ${ }^{8}$
}

To cite: Marsman MS, Wetterslev J, Vriens PW.H.E, et al. Eversion technique versus conventional endarterectomy with patch angioplasty in carotid surgery: protocol for a systematic review with metaanalyses and trial sequential analysis of randomised clinical trials. BMJ Open 2020;10:e030503. doi:10.1136/ bmjopen-2019-030503

- Prepublication history and additional material for this paper are available online. To view these files, please visit the journal online (http://dx.doi. org/10.1136/bmjopen-2019030503).

Received 17 March 2019 Revised 12 November 2019 Accepted 20 November 2019

Check for updates

(C) Author(s) (or their employer(s)) 2020. Re-use permitted under CC BY-NC. No commercial re-use. See rights and permissions. Published by BMJ.

For numbered affiliations see end of article.

Correspondence to

Martijn S. Marsman; mmarsman@rijnstate.nl

\section{ABSTRACT}

Introduction Traditional carotid endarterectomy is considered to be the standard technique for prevention of a new stroke in patients with a symptomatic carotid stenosis. Use of patch angioplasty to restore the arterial wall after longitudinal endarterectomy is, to date, not unequivocally proven to be superior to eversion technique. A systematic review is needed for evaluation of benefits and harms of the eversion technique versus the traditional endarterectomy with patch angioplasty in patients with symptomatic carotid stenosis.

Methods and outcomes The review will be conducted according to this protocol following the recommendations of the 'Cochrane Handbook for Systematic Reviews' and reported according to Preferred Reporting Items for Systematic Reviews and Meta-Analyses. Randomised clinical trials comparing eversion technique versus endarterectomy with patch angioplasty in patients with a symptomatic stenosis of the internal carotid artery will be included. Primary outcomes are all-cause mortality rate, health-related quality of life and serious adverse events. Secondary outcomes are 30-day stroke and mortality rate, symptomatic arterial restenosis or occlusion and non-serious adverse events. The databases Cochrane Central Register of Controlled Trials, PubMed/MEDLINE and EMBASE will be searched (November 2019). We will primarily base our conclusions on meta-analyses of trials with overall low-risk of bias. We will use trial sequential analysis to assist the evaluation of imprecision in Grading of Recommendations, Assessment, Development and Evaluation. However, if pooled point estimates of all trials are similar to pooled point estimates of trials with overall low risk of bias and there is lack of a statistical significant interaction between estimates from trials with overall high risk of bias and trials with overall low risk of bias we will consider the trial sequential analysis adjusted precision of the estimate achieved in all trials as the result of our meta-analyses.

Ethics and dissemination The proposed systematic review will collect and analyse data from published
Strengths and limitations of this study

- The review shall be conducted according to this published protocol following the recommendations of the 'Cochrane Handbook for Systematic Reviews of Interventions' and reported according to the Preferred Reporting Items for Systematic Reviews and Meta-Analyses statement.

- Trial sequential analysis (TSA) compared with Grading of Recommendations, Assessment, Development and Evaluation assessments of randomised clinical trials is included.

- This review benefits from a comprehensive search strategy, designed to retrieve a broad spectrum of relevant articles for the research question.

- To reduce chance for design error in interventions, one technique (the experimental intervention, the eversion technique) will be compared with one other technique (the control intervention, the traditional carotid endarterectomy with patch angioplasty).

- Depending on the number of included randomised clinical trials firm conclusions based the TSA analysis can possibly not be made.

studies, therefore, ethical approval is not required. The results of the review will be disseminated by publication in a peer-review journal and submitted for presentation at conferences.

PROSPERO registration number CRD42019119361.

\section{INTRODUCTION}

Carotid artery stenosis occurs due to atherosclerosis and was described to be a pathological substrate for ischaemic diseases of the ipsilateral brain and eye by Fisher. ${ }^{1}$ Preventive management of asymptomatic carotid artery stenosis includes antiplatelet, statins, 
antihypertensive medication, diabetic control, as well as lifestyle modifications. ${ }^{2-4}$ Traditional carotid endarterectomy (tCEA) is the preferred guideline treatment for patients with symptomatic stenosis of the carotid artery, ${ }^{56}$ primarily based on the European Carotid Surgery Trial and the North American Symptomatic Carotid Endarterectomy Trial. ${ }^{7-9}$

Two operation techniques are used globally: the eversion technique (ET) and the tCEA using a longitudinal arteriotomy and patch angioplasty. Both techniques have the same approach to the carotid artery. ET was first reported by De Bakey et al and later described by Etheredge. ${ }^{10}$ This technique has a potential advantage compared with the tCEA, because patch closure is not always necessary, but the downside is the possibility of transection of carotid sinus nerve branches resulting in loss of the baroreceptor reflex. Whereas tCEA using a longitudinal arteriotomy, the incision is made parallel to the nerve branches, with smaller chance of transection of these nerve fibres. Loss of the baroreceptor reflex is associated with postoperative hypertension, a risk factor for cerebral hyper perfusion syndrome. The sympathetic trunk is another structure at risk, damage may result in signs of Horner's syndrome.

Closure in both techniques can be achieved by either direct suturing of the arterial wall or patch angioplasty in CEA. ${ }^{11}$ TCEA with patch (for closing the longitudinal arteriotomy of the arterial wall) is suggested to reduce both the risks of restenosis and recurrent ipsilateral stroke. ${ }^{12}$ Restenosis after tCEA occurs in 6\%-36\% of patients during long-term follow-up of at least 12 months. ${ }^{13-17}$ Restenosis after ET occurs in $1.7 \%-2.5 \%$ of patients during long-term follow-up of at least 12 months. ${ }^{18}$

European guidelines of both the European Society of Vascular Surgery and the Dutch society for vascular surgery consider CEA with patch angioplasty as the reference technique. ${ }^{91920}$ A meta-analysis of 6 randomised clinical trials (RCTs) including 2790 operations in 2666 patients compared ET with tCEA and concluded that ET may reduce the risks of perioperative stroke and long-term restenosis. ${ }^{21}$ However, the observed differences in intervention effects may be explained by several confounding factors and/or differential use of cointerventions, such as the use of perioperative transcranial Doppler (TCD) monitoring, perioperative carotid pressure measurement, electroencephalographic (EEG) monitoring, selected use of shunting, regional anaesthesia and variations in materials used for patching. ${ }^{22-29}$

To determine which technique, ET or tCEA is more effective for symptomatic carotid stenosis from the patients' perspective, it is important that all available evidence is evaluated according to the risks of errors in a systematic review in line with the Cochrane Handbook for Systematic Reviews of Interventions. ${ }^{30} 31$ Therefore, an updated systematic review with meta-analyses and trial sequential analysis (TSA) is needed including Grading of Recommendations, Assessment, Development and Evaluation (GRADE) assessments of the evidence.
Previous reviews such as the one by Paraskevasa $e t a l^{32}$ could be considered as 'similar' to this review. However, the differences can be found in the methodological approach. Our review will be:

- Conducted after a prepublished protocol.

- We would like to compare ONE technique (eversion CEA) with ONE other technique (CEA with patch closure) to lower the chance of bias (avoid design error).

- We will report numbers of patients with complications instead of the incidence of complications based on number of CEAs.

- Besides performing conventional meta-analysis, we have also planned to perform TSA.

- Conclusions made using TSA (according to the instructions for use ${ }^{33}$ ) may very well be more reliable than those using traditional meta-analysis techniques only.

\section{Objective}

The objective is to conduct a systematic review with metaanalysis and TSA of randomised clinical trials, evaluating the benefits and harms of the ET versus the tCEA in symptomatic patients according to a prepublished protocol following the Cochrane Handbook for Systematic Reviews of Interventions. ${ }^{30}$

\section{METHODS}

This review will be conducted according to this protocol, registered at PROSPERO ${ }^{34}$ following the recommendations of the "Cochrane Handbook for Systematic Reviews of interventions ${ }^{, 30}$ and will be reported according to the Preferred Reporting Items for Systematic Reviews and Meta-Analyses statement (www.prisma-statement.org). ${ }^{35}$

\section{Studies}

Only randomised clinical trials comparing ET versus tCEA with patch angioplasty (regardless of the types of patch materials used) will be included. Trials will be considered irrespective of language, blinding, outcomes or publication status. Two authors will screen all the hits from all the searching machines manually for RCTs without the use of a computerised filter. We will also consider quasirandomised studies, controlled clinical studies and other observational studies for data on harm if retrieved with our searches for randomised clinical trials. So it may occur that trials that are not RCT can be included for data on harm if retrieved. This is because adverse events are rarely reported in randomised clinical trials. ${ }^{36}$ Moreover, observational studies may provide information on rare or late occurring adverse events. ${ }^{36}$ We are aware that the decision not to search for all observational studies may bias our review towards assessment of benefits and may overlook certain harms, such as late or rare harms. However, we will not include observational studies together with RCTs in the meta-analyses. 


\section{Patients}

According to the current guideline ${ }^{7-9}$ patients with a symptomatic stenosis $(>50 \%)$ of the carotid artery will be considered. Only trials which evaluate the ET versus the tCEA in adult patients ( $\geq 18$ years) will be included. ${ }^{20}$ We are aware of the guideline statement that endarterectomy may be considered in symptomatic internal carotid lesions of $<70 \%$. Studies in children and animals will be excluded.

\section{Experimental intervention}

The experimental intervention is the ET. The current ET for resolving the symptomatic stenosis in the (internal) carotid artery is based on the description by Etheredge $\mathrm{e}^{10}$ and involves an oblique circumferential transection of the internal carotid artery (ICA) at the level of the carotid sinus. After distal eversion the diseased intimal plaque is totally excised from the tunica media and tunica adventitia and subsequently after endarterectomy of the carotid sinus, anatomical reimplantation of ICA follows. ${ }^{37}$

We wish to compare one experimental intervention to one control intervention to reduce chance for design error (clinical heterogeneity) in the experimental intervention used. Therefore, RCTs which compare primary closure of the arterial wall after longitudinal arteriotomy will be excluded. Other techniques for carotid surgery in symptomatic patients are investigated in separate reviews. $^{3839}$

\section{Control intervention}

The control intervention is the tCEA with patch closure of the longitudinal incision made in the carotid artery regardless of the type of patch material used. ${ }^{11}$

\section{Cointerventions and cerebral monitoring}

Procedures may either be performed under plexus or general anaesthesia. Patients usually receive 5000 international units of heparin intravenously before crossclamping the carotid artery. Sometimes protamine sulfate is given after surgery. TCD is used for microemboli detection and look for any increase of blood flow to the brain during the surgery. Patients will or can be monitored intraoperatively with EEG. Patients will or can receive a shunt when EEG changes are observed. Intraoperative monitoring may vary in the trials such as the use of perioperative TCD monitoring, perioperative carotid pressure measurement, EEG monitoring. Other intraoperative cointerventions may also vary in the trials, for example, the selected use of shunting and the variations in the types of materials used.

\section{Hypothesis}

We want to relate to the null hypothesis that there is not any difference between the two treatments (H0: relative risk reduction $(\mathrm{RRR})=0.00 \%$ or risk ratio $(\mathrm{RR})=1.00)$ as well as both the alternative hypotheses (H1a and H1b) that there is a difference (H1a of a $10 \%$ RRR or H1b of a $15 \%$ RRR) between ET and tCEA in patients with a symptomatic carotid lesion.

\section{The importance of outcomes}

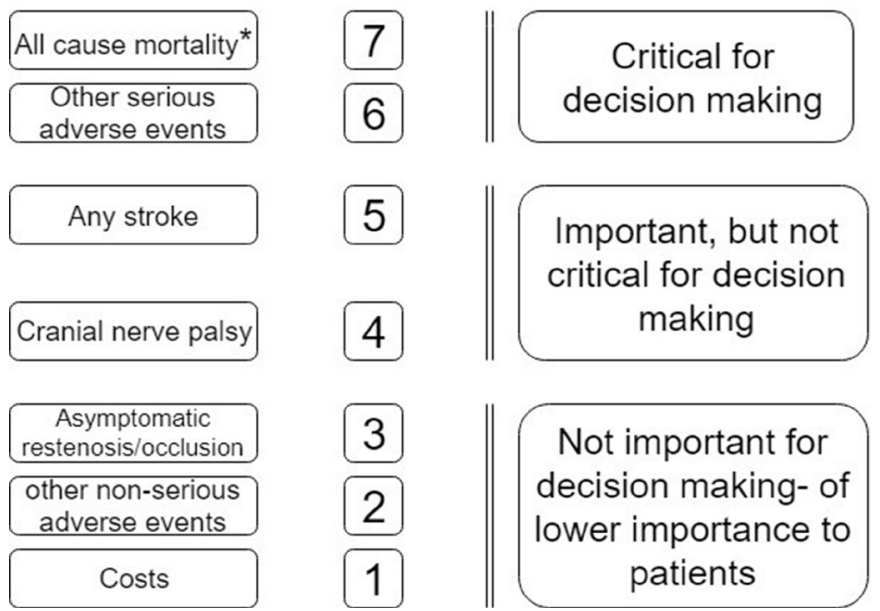

Figure 1 Outcomes prioritised according to importance to patients (critical for descision making) undergoing carotid surgery for symptomatic carotid stenosis (GRADE 2008)..$^{40}$

${ }^{*}<30$ days and long term ( $>30$ days). GRADE, Grading of Recommendations, Assessment, Development and Evaluation.

\section{Outcomes}

The outcome measures will be graded from the patients' perspective (GRADE working group 2008, figure 1). ${ }^{40}$ Examples of serious adverse events (SAEs): stroke, bleeding, persisting neurological deficits, for example, patients developing signs of Horner's syndrome, ${ }^{41}$ hypertension in need for (intravenous) medication.

Stroke and mortality within 30 days were considered as secondary outcomes. These outcomes are important but possibly unreliable especially in surgical interventions as short time outcomes may prevail for one intervention while (with crossing of survival curves) the other intervention prevail on the long term. ${ }^{42}$

\section{Primary outcomes}

- All-cause mortality.

- Proportion of participants with one or more SAEs; which is defined as: any untoward medical occurrence that results in death is life threatening, requires hospitalisation or prolongation of existing hospitalisation, results in persistent or significant disability or incapacity (or is a congenital anomaly or birth defect). ${ }^{43}$

- Health status (or: quality of life): any scale used by trialists to assess the participants' reporting of their quality of life (or health status).

\section{Secondary outcomes}

- <30 days mortality rate.

- <30 days stroke rate.

- Symptomatic (50\%-99\%) arterial restenosis or occlusion.

- Proportion of participants with one or more non-SAEs: any untoward medical occurrence in a participant that 
does not meet the above criteria for a SAE is defined as a non-SAE. ${ }^{43}$

- Lower importance for patients: asymptomatic (50\%$99 \%$ ) arterial restenosis or occlusion.

\section{Exploratory outcomes}

- Separately reported SAEs.

- Separately reported non-SAEs.

A number of patients with one or more complications were evaluated rather than the numbers of events, depending on the availability of data.

\section{Search strategy}

The Cochrane Central Register of Controlled Trials (CENTRAL) in the Cochrane Library, PubMed/ MEDLINE and EMBASE will be searched. References of the identified trials will be searched to identify any further relevant randomised clinical trials. The search strategies are provided in online supplementary appendix 1. Searches will include MeSH descriptors such as 'Clinical Trials', 'carotid endarterectomy', 'eversion', 'carotid artery disease'. We will also search online trial registries such as ClinicalTrials.gov (https://clinicaltrials.gov/), European Medicines Agency (EMA) (www.ema.europa. eu/ema/), WHO International Clinical Trial Registry Platform (www.who.int/ictrp) and the Food and Drug Administration (FDA) (www.fda.gov) for ongoing or unpublished trials. In addition, we plan to search Google Scholar (https://scholar.google.nl/) using the terms: eversion and/or Carotid and/or Endarterectomy in the title of the abstract/paper.

\section{Data collection}

Two authors will perform screening and select the trials for inclusion, independently. Excluded trials and studies will be listed with their reasons for exclusion. When disagreements should occur, a third author will be approached to reconcile. The authors will extract the following data: trial characteristics (year and language of publication, country in which the trial was conducted, year of conduction of the trial, single or multicentre trial, number of patients), patient characteristics (inclusion and exclusion criteria, mean age, mean body mass index and gender, smoking, diabetes mellitus, use of statin and platelet inhibitors), intervention characteristics (primary closure, closure by patch, use of shunting), cointerventions (local or general anaesthesia, perioperative TCD monitoring, perioperative carotid pressure measurement, EEG monitoring) and the outcome measures evaluated. If there are any unclear or missing data, the corresponding authors of the individual trials will be contacted, at least twice, for clarification.

\section{Risk of bias assessment}

Two authors will assess the risks of bias, without masking for trial names, according to the Cochrane Handbook for Systematic Reviews of Interventions, ${ }^{30}$ including the domains of generation of the allocation sequence, allocation concealment, blinding of participants, personnel and outcome assessors, incomplete outcome data, selective outcome reporting and bias risks such as vested interests (financial interest, academical interest or other parties such as the medical industry). Risk of bias components were scored as low, unclear or high risk of bias. Trials were classified as trials with low overall risk of bias if all risk of bias domains were scored as having low risk of bias. If one or more of the bias domains were scored as unclear or high risk of bias, the trial was considered to have high overall risk of bias. Trials classified as low risk of bias in all domains of sequence generation, allocation concealment, blinding, incomplete data, selective outcome reporting, source of funding and other potential risks of bias will be considered trials at overall low risk of bias. Trials with one or more of these domains scored as unclear or high risk of bias will be considered trials at overall high risk of bias. ${ }^{31} 4445$

\section{Sequence generation}

- Low risk of bias: The method used (eg, central allocation) is unlikely to induce bias on the final observed effect, such as:

- Referring to a random number table.

- Using a computer random number generator.

- Coin tossing.

- Shuffling cards or envelopes.

- Throwing a dice.

- Drawing of lots.

- Unclear risk of bias: Insufficient information to assess whether the method used is likely to introduce confounders.

- High risk of bias: The method is improper and likely of introduce confounding, for example, based on date of admission, or record number, or by odd or even date of birth.

\section{Allocation concealment}

Some aspects of the conduct of randomised trials, particularly blinding, are associated with a modest exaggeration of treatment effects on average, but there is little evidence that the average bias differs according to whether the outcome was subjectively or objectively assessed. However, lack of blinding in trials with subjective outcomes leads to increased heterogeneity and hence unpredictable bias in effect estimates. As far as possible, clinical and policy decisions should be cautious when they are based on trials in which blinding was not reported or not feasible and outcome measures were subjectively assessed. ${ }^{45}$

- Low risk of bias: participants and investigators enrolling participants could not foresee assignment because one of the following, or an equivalent method, was used to conceal allocation:

- Central allocation (including telephone).

- Web-based and pharmacy-controlled randomisation.

- Sequentially numbered drug containers of identical appearance.

- Sequentially numbered, opaque, sealed envelopes. 
- Unclear risk of bias: insufficient information to permit judgement of 'low risk' or 'high risk'. This is usually the case if the method of concealment is not described or not described in sufficient detail to allow a definite judgement.

- High risk of bias: participants or investigators enrolling participants could possibly foresee assignments and thus introduce selection bias, such as allocation based on:

- An open random allocation schedule.

- Assignment envelopes were used without appropriate safeguards.

- Alternation or rotation.

- Date of birth.

- Case record number.

- Any other explicitly unconcealed procedure.

\section{Blinding of participants and personnel}

In surgical procedures, it is impossible to blind the surgeon who performs the procedure of CEA, while it is possible to blind the caregivers responsible for postoperative care as well as the patients. ${ }^{46}$ For this domain, we will consider the caregivers and patients and not the surgeon who performs the procedure, although a certain risk of bias will inevitably be present when evaluating surgical procedures.

- Low risk of bias: no blinding or incomplete blinding, but the review authors judge that the outcome is not likely to be influenced by lack of blinding or blinding of participants and key study personnel ensured, and it is unlikely that the blinding could have been broken.

- Unclear risk of bias: insufficient information to permit judgement of 'low risk' or 'high risk', or the study did not address this outcome.

- High risk of bias: no blinding or incomplete blinding, and the outcome is likely to be influenced by lack of blinding or blinding of key study participants and personnel attempted, but likely that the blinding could have been broken, and the outcome is likely to be influenced by lack of blinding.

\section{Blinding of outcome assessment}

- Low risk of bias: no blinding of outcome assessment, but the review authors judge that the outcome measurement is not likely to be influenced by lack of blinding or blinding of outcome assessment is ensured, and it is unlikely that the blinding could have been broken.

- Unclear risk of bias: insufficient information to permit judgement of 'low risk' or 'high risk' or the study did not address this outcome.

- High risk of bias: no blinding of outcome assessment, and the outcome measurement is likely to be influenced by lack of blinding, or blinding of outcome assessment, but likely that the blinding could have been broken, and the outcome measurement is likely to be influenced by lack of blinding.

\section{Incomplete outcome data}

- Low risk of bias:

- No missing outcome data.

- Reasons for missing outcome data unlikely to be related to true outcome (for survival data, censoring unlikely to be introducing bias).

- Missing outcome data balanced in numbers across intervention groups, with similar reasons for missing data across groups.

- For dichotomous outcome data, the proportion of missing outcomes compared with observed event risk is not enough to have a clinically relevant impact on the intervention effect estimate.

- For continuous outcome data, plausible effect size (difference in means or standardised difference in means) among missing outcomes is not enough to have a clinically relevant impact on observed effect size.

- Missing data have been imputed using appropriate methods.

- Unclear risk of bias: insufficient reporting of attrition/exclusions to permit judgement of 'low risk' or 'high risk' (eg, number randomised not stated, no reasons for missing data provided) or the study did not address this outcome.

High risk of bias:

- Reason for missing outcome data likely to be related to true outcome, with either imbalance in numbers or reasons for missing data across intervention groups.

- For dichotomous outcome data, the proportion of missing outcomes compared with observed event risk enough to induce clinically relevant bias in intervention effect estimate.

- For continuous outcome data, plausible effect size (difference in means or standardised difference in means) among missing outcomes enough to induce clinically relevant bias in observed effect size.

- 'As-treated' analysis done with substantial departure of the intervention received from that assigned at randomisation.

- Potentially inappropriate application of simple imputation.

\section{Selective outcome reporting}

- Low risk of bias: the study protocol is available and all the studies prespecified (primary and secondary) outcomes that are of interest in the review have been reported in the prespecified way, or the study protocol is not available but it is clear that the published reports include all expected outcomes, including those that were prespecified.

- Unclear risk of bias: insufficient information to permit judgement of 'low risk' or 'high risk'. It is likely that the majority of studies will fall into this category.

- High risk of bias:

- Not all of the studies prespecified primary outcomes have been reported. 
- One or more primary outcomes is reported using measurements, analysis methods or subsets of the data (eg, subscales) that were not prespecified.

- One or more reported primary outcomes were not prespecified (unless clear justification for their reporting is provided, such as an unexpected adverse effect).

- One or more outcomes of interest in the review are reported incompletely so that they cannot be entered in a meta-analysis.

- The study report fails to include results for a key outcome that would be expected to have been reported for such a study.

\section{Other bias}

- Low risk of bias: the study appears to be free of other sources of bias.

- Unclear risk of bias: there may be a risk of bias, but there is either insufficient information to assess whether an important risk of bias exists or insufficient rationale or evidence that an identified problem will introduce bias.

- High risk of bias: there is at least one important risk of bias.

\section{Statistical methods}

Meta-analyses will be performed according to the Cochrane Handbook for Systematic Reviews of Interventions. ${ }^{30}$ The software package Review Manager (RevMan) V.5.3 will be used. ${ }^{47}$ Significance levels will be adjusted due to multiplicity of several outcomes. The results of each outcome will require an adjusted statistical significance level (threshold). An alpha of $(0.05 /((1+3) / 2)=)$ 0.025 will be used for the primary outcomes to keep the family wise error rate (FWER) below 0.05 . For the secondary outcomes, this will be $0.017 .^{48}{ }^{49}$ For exploratory outcomes, we will consider a $p<0.05$ as significant, because we view these outcomes as only hypothesisgenerating outcomes. For dichotomous variables, the RR with TSA-adjusted CIs will be calculated. For continuous variables, the mean difference (MD) or the standardised MD with $95 \%$ CI will be calculated.

For the outcome of SAE, we plan to estimate the proportion of patients with one or more SAE in each group and to analyse this outcome in a binary meta-analysis. However, as we anticipate the reporting of SAEs in trials to vary considerably we plan to do two analyses:

1. The cumulated SAE analysis: Assuming that only one SAE is reported per patient. We will summarise all reported SAE in each trial and calculate the proportion of summed SAE divided with number of randomised patients in the experimental and control intervention group, the number of patients in each group will be a maximum.

2. To avoid multiple counts of SAE in the same patients (SAE counting is not a statistical independent outcome) we will also analyse the most frequent SAE as if it represents the total number of SAEs in the experimental and control intervention group (best case scenario). Being aware that none of these intervention effect estimates are exactly correct we will discuss differences between the effect of the experimental versus the control intervention on the proportion of patients with one or more SAEs.

The impact of attrition bias will be explored using best/ worst and worst/best-case scenarios: a best/worst-case scenario is one where all patients lost to follow-up in the intervention group are supposed to have survived while all patients lost to follow-up in the control intervention group have died. A worst/best-case scenario is the reverse.

Heterogeneity will be explored by $\mathrm{X}^{2}$ test with significance set at $p$ value of 0.10 , and the quantity of heterogeneity will be measured by $\mathrm{I}^{2}$. We will conduct both random-effects model and fixed-effect model metaanalyses. In case of discrepancies, the results of both models will be presented and we will primarily stress the result of the model with the result closest to null effect due to the principle of cautiousness. ${ }^{49}$ The analyses will be performed on an intention-to-treat basis whenever possible.

A funnel plot will be used to explore small trial bias and to use asymmetry in funnel plot of trial size against treatment effect to assess this bias. Begg's and Egger's tests will be used to test for asymmetry in funnel plots. ${ }^{50}$

\section{Trial sequential analyses}

Meta-analyses may result in type I errors and type II errors due to an increased risk of random error when sparse data are analysed and due to repeated significance testing when a cumulative meta-analysis is updated with new trials. ${ }^{5152}$ To assess the risk of type I and type II errors, TSA will be used. The vast majority of meta analyses (nearly $80 \%$ ) in Cochrane systematic reviews have less than the required information size to conclude on a 30\% RRR and less than $2 \%$ have sufficient power to conclude on a $10 \%$ RRR. ${ }^{53-55}$

TSA combines information size estimation for metaanalysis (cumulated sample size of included trials) with an adjusted threshold for statistical significance of metaanalysis. ${ }^{51} 5256$ The latter, called trial sequential monitoring boundaries (TSMBs), reduce type I errors. In TSA, the addition of each trial in a cumulative meta-analysis is regarded as an interim analysis and helps to clarify whether additional trials are needed or not. The idea in TSA is that when the cumulative z-curve crosses the TSMB, a sufficient level of evidence has been reached and no further trials may be needed. If the z-curve does not cross the boundary of benefit and the required information size has not been reached, there may be insufficient evidence to reach a conclusion. ${ }^{51525758}$ TSA can also be used for the evaluation of type II errors, that is, to evaluate whether further randomised trials are futile to show or discard the anticipated intervention effect (RRR or MD). This happens when the cumulative z-curve does cross the TSMBs for futility. TSA will be applied since it controls the risks of type I and type II errors in a cumulative metaanalysis and may provide important information on 
how many more patients need to be included in further trials. The information size will be calculated as diversityadjusted required information size (DARIS).$^{59}$ We will do the primary analysis calculating the DARIS based on an a priori anticipated intervention effect of a 10\% RRR which is close to a minimal important difference. We will conduct sensitivity analyses for a 15\% RRR as well as a the RRR suggested by the meta-analysis of the included trials. ${ }^{60}$ If the estimated Diversity of the meta-analysis is $0 \%$, a sensitivity analysis with TSA using a diversity of $25 \%$ will be conducted. TSA will be performed on all outcomes. The required information size for primary outcomes will be calculated based on an a priori RRR of $10 \%$ and appropriately adjusted for diversity according to an overall type I error of $2.5 \%$ for the coprimary outcomes and $1,7 \%$ for the secondary outcomes to account for a FWER of $5 \%$ all in all, we will use a power of $90 \%$ considering sparse data and repetitive testing. ${ }^{59}$ For secondary outcomes, the DARIS will be calculated using a power of $90 \%{ }^{59}$ As a sensitivity analysis, the DARIS will be calculated using the estimated intervention effect from the trials at low risk of bias in a conventional meta-analysis. If the required information size is surpassed for the TSA using the estimated intervention effect in the conventional meta-analysis or a TSMB is crossed a TSA with an anticipated intervention effect equal to the confidence limit closest to the null effect in the effect estimate from the conventional metaanalysis will be performed. The TSAs will be conducted using the control event proportion calculated from the unweighted control event proportion from the control groups of the actual meta-analyses.

\section{Subgroup analyses}

The following subgroup analyses will be performed:

Trials at overall low risk of bias (all except blinding of surgeons scored as low risk of bias) compared with trials at high overall risk of bias (two or more of the bias domains (excluding blinding of surgeons) scored as unclear or high risk).

Different patch materials may be used including venous, polytetrafluorethylene, Dacron and biopatches (bovine/porcine) ${ }^{29}$ Subgroup analyses will be conducted according availability of data on different types of materials.

\section{Grading of Recommendations, Assessment, Development and Evaluation}

We will use summary of findings tables to summarise the results of the trials with overall low risk of bias and for all trials, separately. Reasons for downgrading the quality of the available evidence are: risk of bias evaluation of the included bias domains, publication bias, heterogeneity, imprecision and indirectness (eg, length of stay is a surrogate outcome measure) ${ }^{61-63}$ We will compare the imprecision assessed according to GRADE with that of TSA. ${ }^{64}$

\section{Patient and public involvement}

Patients and/or public were not involved in this study.

\section{Ethics and dissemination}

The results of the systematic review will be disseminated by publication in a peer-review journal and submitted for presentation at relevant conferences. This protocol will be online available prior to the start of the review process, and at the PROSPERO website. ${ }^{34}$

\section{Resemblances in literature}

Our previous published protocol ${ }^{39}$ may show overlap with this current protocol. This overlap is because of the basic methods to conduct a review process in line with the Cochrane Handbook. ${ }^{30}$ Nevertheless, this review topic differs on specific important details for the well informed vascular community. We would like to emphasise that it is important to compare ONE (experimental) technique with ONE other technique (control intervention) to reduce the chance for design error. In many other reviews, all kinds of techniques are compared with other more or less similar techniques. This is wrong. The important point (one-to-one technique comparisons) was recently highlighted during a global Vascular Congress (VEITH) November 2019, New York, USA. Together with this important point, the rigid methodological approach and the sophisticated and specific recommendations were followed during the design process of this protocol. Together with the Copenhagen Trial Unit, we managed to reach consensus and a detailed description of specific outcomes. To some readers, this may only look as if there is a lot of duplication. However, the differences are in the details and are specific and needed to proceed in evaluating important techniques to reduce complication rates.

\section{Author affiliations}

${ }^{1}$ Department of Vascular Surgery, Rijnstate Hospital, Arnhem, The Netherlands ${ }^{2}$ Rigshospitalet, Copenhagen Trial Unit, Centre for Clinical Intervention Research, Copenhagen, Denmark

${ }^{3}$ Department of Vascular Surgery, Elisabeth-Tweesteden Hospital, Tilburg, The Netherlands

${ }^{4}$ Department of Anatomy, University Medical Center Utrecht, Utrecht, The Netherlands

${ }^{5}$ Department of Vascular Surgery, HFG, Medical Center Leeuwarden, Leeuwarden, The Netherlands

${ }^{6}$ Department of Vascular Surgery, University Medical Center Utrecht, Utrecht, The Netherlands

${ }^{7}$ Department of Critical Care, University of Groningen, University Medical Center Groningen, Groningen, The Netherlands

${ }^{8}$ Department of Vascular Surgery, Ikazia Hospital, Rotterdam, The Netherlands

Acknowledgements The authors would like to thank LWM. Boerboom, medical information specialist (Medical Library, Elisabeth-TweeSteden Hospital, Tilburg, the Netherlands) for her assistance.

Contributors MSM is the first author of the protocol. MSM and GGK managed the first draft of this manuscript and coordinated the contributions of coauthors. JW, $\mathrm{PV}, \mathrm{RB}, \mathrm{AKJ}, \mathrm{FLM}, \mathrm{FK}$ and GGK contributed to the design of the study and revised the paper critically. JW, FK and GGK provided professional and statistical support. All authors read and approved the final version of the manuscript. GGK was initiator and supervisor.

Funding The authors have not declared a specific grant for this research from any funding agency in the public, commercial or not-for-profit sectors.

Competing interests JW is a member of the taskforce at Copenhagen Trial Unit to develop theory and software doing TSA, presently available as freeware at www. ctu.dk/tsa. 
Patient consent for publication Not required.

Provenance and peer review Not commissioned; externally peer reviewed.

Open access This is an open access article distributed in accordance with the Creative Commons Attribution Non Commercial (CC BY-NC 4.0) license, which permits others to distribute, remix, adapt, build upon this work non-commercially, and license their derivative works on different terms, provided the original work is properly cited, appropriate credit is given, any changes made indicated, and the use is non-commercial. See: http://creativecommons.org/licenses/by-nc/4.0/.

\section{ORCID iDs}

Martijn S. Marsman http://orcid.org/0000-0002-9819-8080

Jorn Wetterslev http://orcid.org/0000-0001-7778-1771

\section{REFERENCES}

1 Fisher M. Occlusion of the internal carotid artery. Arch Neurol Psychiatry 1951;65:346-77.

2 Raman G, Moorthy D, Hadar N, et al. Management strategies for asymptomatic carotid stenosis: a systematic review and metaanalysis. Ann Intern Med 2013;158:676-85.

3 Abbott AL. Medical (nonsurgical) intervention alone is now best for prevention of stroke associated with asymptomatic severe carotid stenosis: results of a systematic review and analysis. Stroke 2009;40:e573-84.

4 Constantinou J, Jayia P, Hamilton G. Best evidence for medical therapy for carotid artery stenosis. J Vasc Surg 2013;58:1129-39.

5 Orrapin S, Rerkasem K, Cina CS. Carotid endarterectomy for symptomatic carotid stenosis. Cochrane Database Syst Rev 2017;6:CD001081.

6 Bangalore S, Kumar S, Wetterslev J, et al. Carotid artery stenting vs carotid endarterectomy: meta-analysis and diversity-adjusted trial sequential analysis of randomized trials. Arch Neurol 2011;68:172-84.

7 Warlow C. MRC European Carotid Surgery Trial: interim results for symptomatic patients with severe $(70-99 \%)$ or with mild $(0-29 \%)$ carotid stenosis. The Lancet 1991;337:1235-43.

8 Barnett HJM, Taylor DW, Haynes RB, et al. Beneficial effect of carotid endarterectomy in symptomatic patients with high-grade carotid stenosis. N Engl J Med 1991;325:445-53.

9 Naylor AR, Ricco J-B, de Borst GJ, et al. Editor's Choice Management of Atherosclerotic Carotid and Vertebral Artery Disease: 2017 Clinical Practice Guidelines of the European Society for Vascular Surgery (ESVS). Eur J Vasc Endovasc Surg 2018;55:3-81.

10 Etheredge SN. A simple technic for carotid endarterectomy. Am J Surg 1970;120:275-8.

11 De Bakey ME, Crawford ES, Cooley DA, et al. Surgical considerations of occlusive disease of innominate, carotid, subclavian, and vertebral arteries. Ann Surg 1959;149:690-710.

12 Rerkasem K, Rothwell PM. Patch angioplasty versus primary closure for carotid endarterectomy. Cochrane Database Syst Rev 2009:CD000160.

13 Bernstein EF, Torem S, Dilley RB. Does carotid restenosis predict an increased risk of late symptoms, stroke, or death? Ann Surg 1990;212:629-36.

14 Knudsen L, Sillesen H, Schroeder T, et al. Eight to ten years followup after carotid endarterectomy: clinical evaluation and Doppler examination of patients operated on between 1978-1980. Eur J Vasc Surg 1990;4:259-64.

15 Ouriel K, Green RM. Clinical and technical factors influencing recurrent carotid stenosis and occlusion after endarterectomy. $J$ Vasc Surg 1987;5:702-6.

16 Volteas N, Labropoulos N, Leon M, et al. Risk factors associated with recurrent carotid stenosis. Int Angiol 1994;13:143-7.

17 Zierler RE, Bandyk DF, Thiele BL, et al. Carotid artery stenosis following endarterectomy. Arch Surg 1982;117:1408-15.

18 Cao P, De Rango P, Zannetti S. Eversion vs conventional carotid endarterectomy: a systematic review. Eur J Vasc Endovasc Surg 2002;23:195-201.

19 Liapis CD, Bell PRF, Mikhailidis D, et al. ESVS guidelines. invasive treatment for carotid stenosis: indications, techniques. Eur J Vasc Endovasc Surg 2009;37:1-19.

20 Beroerte, Kwaliteitsinstituut voor de Gezondheidszorg CBO. Diagnostiek, behandeling en zorg voor patiënten Met Een beroerte. Nederlandse Vereniging voor Neurologie, 2008. http://med-info.nl/ Richtlijnen/Geriatrie/Beroerte.pdf

21 Antonopoulos CN, Kakisis JD, Sergentanis TN, et al. Eversion versus conventional carotid endarterectomy: a meta-analysis of randomised and non-randomised studies. Eur J Vasc Endovasc Surg 2011;42:751-65.

22 Bass A, Krupski WC, Schneider PA, et al. Intraoperative transcranial Doppler: limitations of the method. J Vasc Surg 1989;10:549-53.

23 Gnanadev DA, Wang N, Comunale FL, et al. Carotid artery stump pressure: how reliable is it in predicting the need for a shunt? Ann Vasc Surg 1989;3:313-7.

24 Kresowik TF, Worsey MJ, Khoury MD, et al. Limitations of electroencephalographic monitoring in the detection of cerebral ischemia accompanying carotid endarterectomy. J Vasc Surg 1991;13:439-43.

25 Kearse LA, Brown EN, McPeck K. Somatosensory evoked potentials sensitivity relative to electroencephalography for cerebral ischemia during carotid endarterectomy. Stroke 1992;23:498-505.

26 Benjamin ME, Silva MB, Watt C, et al. Awake patient monitoring to determine the need for shunting during carotid endarterectomy. Surgery 1993;114:673-9. discussion 679-681.

27 Rerkasem K, Bond R, Rothwell PM. Local versus general anaesthesia for carotid endarterectomy (review). Cochrane Database Syst Rev 2005:CD000126.

28 Rerkasem K, Rothwell PM. Routine or selective carotid artery shunting for carotid endarterectomy (and different methods of monitoring in selective shunting). Cochrane Database Syst Rev 2009:CD000190.

29 Rerkasem K, Rothwell PM, Cochrane Stroke Group. Patches of different types for carotid patch angioplasty. Cochrane Database Syst Rev 2010;24.

30 Higgins J, Green S. Cochrane handbook for systematic review of intervention version 5.1.0 [Internet]. The Cochrane Collaboration, 2011. Available: www.Cochrane-handbook.org

31 Keus F, Wetterslev J, Gluud C, et al. Evidence at a glance: error matrix approach for overviewing available evidence. BMC Med Res Methodol 2010;10:90.

32 Paraskevas KI, Robertson V, Saratzis AN, et al. Editor's Choice An Updated Systematic Review and Meta-analysis of Outcomes Following Eversion vs. Conventional Carotid Endarterectomy in Randomised Controlled Trials and Observational Studies. Eur J Vasc Endovasc Surg 2018;55:465-73.

33 Copenhagen Trial Unit. Trial Sequential Analysis software [Internet]. Available: http://www.ctu.dk/tsa

34 Marsman MS, Wetterslev J, Vriens $\mathrm{P}$, et al. Eversion technique versus conventional endarterectomy with patch angioplasty in carotid surgery: protocol for a systematic review with metaanalyses and trial sequential analysis of randomized clinical trials. PROSPERO 2019 CRD42019119361, 2019. Available: http://www.crd.york.ac.uk/PROSPERO/display_record.php?ID= CRD42019119361

35 Moher D, Liberati A, Tetzlaff J, et al. Preferred reporting items for systematic reviews and meta-analyses: the PRISMA statement. PLoS Med 2009;6:e1000097.

36 Storebø OJ, Pedersen N, Ramstad E, et al. Methylphenidate for attention deficit hyperactivity disorder (ADHD) in children and adolescents - assessment of adverse events in non-randomised studies. Cochrane Database Syst Rev 2018;5:CD012069.

37 Djedovic M, Mujanovic E, Hadzimehmedagic A, et al. Comparison of results classical and eversion carotid endarterectomy. Med Arch 2017;71:89.

38 Marsman MS, Wetterslev J, AKh J, et al. Carotid endarterectomy with primary closure versus patch angioplasty in patients with symptomatic and significant stenosis: protocol for a systematic review with meta-analyses and trial sequential analysis of randomized clinical trials [Internet], 2018. Available: https://www.crd. york.ac.uk/prospero/display_record.php?RecordID=13416 [Accessed 17 Oct 2018].

39 Marsman MS, Wetterslev J, Jahrome AK, et al. Carotid endarterectomy with primary closure versus patch angioplasty in patients with symptomatic and significant stenosis: protocol for a systematic review with meta-analyses and trial sequential analysis of randomised clinical trials. BMJ Open 2019;9:e026419.

40 Guyatt GH, Oxman AD, Kunz R, et al. What is "quality of evidence" and why is it important to clinicians? BMJ 2008;336:995-8.

41 Koning GG, Lüning TH, ter Bruggen J. Een vrouw Met linkszijdig retrobulbaire pijn, fotofobie en hornersyndroom. Ned Tijdschr voor Geneeskd 2007;10:55-7.

42 Stone GW, Kappetein AP, Sabik JF, et al. Five-Year outcomes after PCl or CABG for left main coronary disease. N Engl J Med 2019;381:1820-30.

43 International Conference on Harmonisation Expert Working Group. International Conference on harmonization of technical requirements for registration of pharmaceuticals for human use. $\mathrm{ICH}$ harmonised tripartite guideline. guideline for good clinical practice 1997;1. 
44 Higgins JPT, Lasserson T, Chandler J, et al. Methodological expectations of Cochrane intervention reviews (MECIR) project. London: Cochrane methods, 2012: 2-3.

45 Savović J, Turner RM, Mawdsley D, et al. Association between Riskof-Bias assessments and results of randomized trials in Cochrane reviews: the ROBES Meta-Epidemiologic study. Am J Epidemiol 2018;187:1113-22.

46 Gurusamy KS, Gluud C, Nikolova D, et al. Assessment of risk of bias in randomized clinical trials in surgery. $\mathrm{Br} J$ Surg 2009;96:342-9.

47 Cochrane Community. Review Manager (RevMan) [Computer program]. Version 5.3. Copenhagen: The Nordic Cochrane Centre, The Cochrane Collaboration, 2014. https://community.cochrane.org/ help/tools-and-software/revman-5

48 Jakobsen JC, Wetterslev J, Lange T, et al. Editorial-Viewpoint: taking into account risks of random errors when analysing multiple outcomes in systematic reviews. Cochrane Database Syst Rev 2016;3:ED000111.

49 Jakobsen JC, Wetterslev J, Winkel P, et al. Thresholds for statistical and clinical significance in systematic reviews with meta-analytic methods. BMC Med Res Methodol 2014;14:1-13.

50 Koning GG, Wetterslev J, van Laarhoven CJHM, et al. Correction: the totally extraperitoneal method versus Lichtenstein's technique for inguinal hernia repair: a systematic review with meta-analyses and trial sequential analyses of randomized clinical trials. PLoS One 2013;8.

51 Wetterslev J, Thorlund K, Brok J, et al. Trial sequential analysis may establish when firm evidence is reached in cumulative meta-analysis. J Clin Epidemiol 2008;61:64-75.

52 Brok J, Thorlund K, Wetterslev J, et al. Apparently conclusive metaanalyses may be inconclusive--Trial sequential analysis adjustment of random error risk due to repetitive testing of accumulating data in apparently conclusive neonatal meta-analyses. Int $\mathrm{J}$ Epidemiol 2009;38:287-98.
53 Turner RM, Bird SM, Higgins JPT. The impact of study size on metaanalyses: examination of underpowered studies in Cochrane reviews. PLoS One 2013;8:e59202-8.

54 Mascha EJ. Alpha, beta, meta: guidelines for assessing power and type I error in meta-analyses. Anesth Analg 2015;121:1430-3.

55 Imberger G, Thorlund K, Gluud C, et al. False-Positive findings in Cochrane meta-analyses with and without application of trial sequential analysis: an empirical review. BMJ Open 2016;6:e011890.

56 Thorlund K, Devereaux PJ, Wetterslev J, et al. Can trial sequential monitoring boundaries reduce spurious inferences from metaanalyses? Int J Epidemiol 2009;38:276-86.

57 Pogue J, Yusuf S. Overcoming the limitations of current metaanalysis of randomised controlled trials. Lancet 1998;351:47-52.

58 Pogue JM, Yusuf S. Cumulating evidence from randomized trials: utilizing sequential monitoring boundaries for cumulative metaanalysis. Control Clin Trials 1997;18:580-93.

59 Wetterslev J, Thorlund K, Brok J, et al. Estimating required information size by quantifying diversity in random-effects model meta-analyses. BMC Med Res Methodol 2009;9:1-12.

60 Wetterslev J, Jakobsen JC, Gluud C. Trial sequential analysis in systematic reviews with meta-analysis. BMC Med Res Methodol 2017;17:1-18.

61 Guyatt GH, Oxman AD, Kunz R, et al. GRADE guidelines 6. Rating the quality of evidence--imprecision. J Clin Epidemiol 2011;64:1283-93.

62 Savović J, Jones H, Altman D, et al. Influence of reported study design characteristics on intervention effect estimates from randomised controlled trials: combined analysis of metaepidemiological studies. Health Technol Assess 2012;16:1-81.

63 Savović J, Jones HE, Altman DG, et al. Influence of reported study design characteristics on intervention effect estimates from randomized, controlled trials. Ann Intern Med 2012;157:429-38.

64 Castellini G, Bruschettini M, Gianola S, et al. Assessing imprecision in Cochrane systematic reviews: a comparison of GRADE and trial sequential analysis. Syst Rev 2018;7:1-10. 